\title{
Implementation of Atomic Resolution Electron Tomography of a Needle Sample.
}

M.C. Scott ${ }^{1}$, W. Theis ${ }^{2}$, Rui Xu ${ }^{1}, \mathrm{Li} \mathrm{Wu}^{1}$, Chien-Chun Chen ${ }^{1}$, Colin Ophus ${ }^{3}$, Peter Ercius ${ }^{3}$ and Jianwei $\mathrm{Miao}^{1}$

1. Department of Physics \& Astronomy and California NanoSystems Institute, and University of California, Los Angeles, CA 90095, USA.

2. Nanoscale Physics Research Laboratory, School of Physics and Astronomy, University of Birmingham, Edgbaston, Birmingham B15 2TT, UK.

3. National Center for Electron Microscopy, Molecular Foundry, Lawrence Berkeley National Laboratory, Berkeley, California 94720, USA.

Electron tomography has become a ubiquitous technique for three-dimensional (3D) characterization in the physical and life sciences. In materials science, improved stability and resolution in scanning transmission electron microscopy (STEM) have made this imaging configuration preferable for tomography of nanocrystals. Recently, lattice and crystalline grain structure [1] and atomic resolution defect structures [2] of nanoparticles have been reconstructed in 3D from a tilt series of annular dark field (ADF)-STEM images obtained on an uncorrected Titan STEM. 3D resolution can be further improved by use of an aberration corrected microscope, as was recently demonstrated in the atomic resolution reconstruction of FePt nanoparticles [3]. Reconstructions of FePt particles from data obtained on the TEAM I microscope at Lawrence Berkeley National lab not only resolved most of the atoms in the particles, but also differentiated between the two atomic species in the particles [3]. So far, these high resolution 3D reconstructions of nanoparticles, reconstructed from data with a single tilt axis.

Experimental difficulties that limit resolution in single tilt tomography include the so-called "missing wedge"- a wedge of missing information that arises from the fact that the sample cannot be tilted a full $\pm 90^{\circ}$ due to the geometry of most sample holders. Additionally, to image nanoparticles as described above, the sample is suspended on a substrate, which contributes additional signal that negatively impacts signal to noise ratio and image resolution, especially at high tilt angles. Equally sloped tomography (EST) is an iterative, Fourier-based algorithm that uses equally sloped angular increments, rather than equal angle as is more common in tilt axis tomography. EST, which was originally conceived for phase retrieval in coherent X-ray tomography, can recover missing information, and can also accurately arrive at a solution even with noisy input data [1, 2, 4]. EST makes no assumptions about a sample's crystalline structure or symmetry, and therefore it is possible obtain local atomic resolution information in $3 \mathrm{D}$ with this technique.

Although the effects of the missing wedge of information and loss of image quality due to the substrate can be alleviated by advanced reconstruction algorithms such as EST, these two major drawbacks will always negatively affect resolution in tilt axis tomography. However, difficulties can be removed by changing the sample geometry to a needle shape and rotating about the axis of the needle. By employing the TEAM stage, a custom piezo-controlled 5-axis (x, y, z, $\alpha$ and $\gamma$ ) tilt stage, a needle sample can be rotated a full $\pm 180^{\circ}$, and by varying both $\alpha$ and $\gamma$, an arbitrary rotation axis can be chosen. The TEAM stage, therefore, can deliver not only superior vibrational stability, but also can access tilt geometries 
unavailable to traditional single tilt sample holders [5].

A tungsten needle sample with tip diameter of $<10 \mathrm{~nm}$ was prepared by electrochemical etching and then mounted in a holder appropriate for the TEAM stage. The tip was imaged in ADF-STEM mode in the TEAM I. To collect the data, custom scripting software was used to calculate the appropriate set of tilt angles to rotate about the desired axis, and to roughly center and focus while delivering minimal electron dose. A set of equally sloped angles was calculated such that the tip was rotated about the [011] direction during the dataset. At each angle, two images were taken. Linear sample drift and scan distortion were corrected before reconstruction via EST. Fig 1 shows the $0^{\circ}$ image of the dataset.

By removing the missing wedge and background contribution from the substrate, reconstructions of the tungsten needle show improvement over similar datasets obtained in a single tilt geometry. Although the tungsten tip was reconstructed from only 62 projections, the resolution and contrast of the resultant reconstruction are greatly improved, even compared to reconstructions from single tilt tomography of nanoparticles with more projections and a higher electron dose. Additionally, the sample geometry in this experiment is similar to that of atom probe tomography, and therefore this technique can be applied to a wide variety of materials.

[1] M.C. Scott et al, Nature 483 (2012), p. 444-447

[2] C.-C. Chen et al, Nature 496 (2013), p. 74-77

[3] M.C. Scott et al, Microscopy and Microanalysis 20 (2014), p. 804-805

[4] C. Zhu et al, Phys. Rev. B 88 (2013), p. 100201

[5] P. Ercius et al, Microscopy and Microanalysis 18 (2012), p. 676-683

[6] This work was mainly supported by the U.S. Department of Energy (Grant No. DEFG02-13ER46943). This work was also partially supported by NSF (DMR-1437263) and Office of Naval Research (N00014-14-1-0675). Experiments were performed at the Molecular Foundry, which is supported by the Office of Science, Office of Basic Energy Sciences of the U.S. Department of Energy under Contract No. DE-AC02-05CH11231.

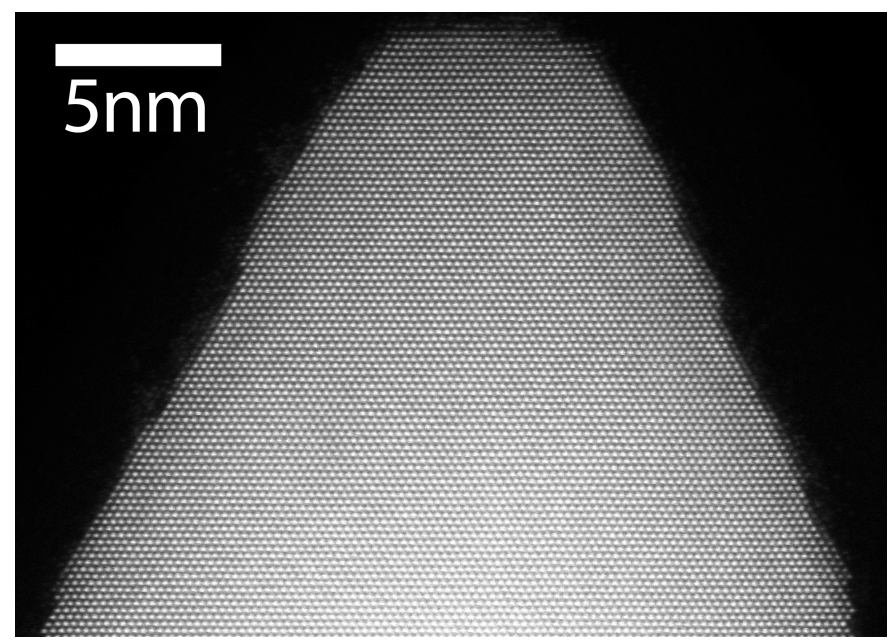

Figure 1. ADF-STEM image of a sharp tungsten tip, imaged along the [111] direction. 